\title{
Introducing a Combined Pattern to Empower Risk Assessment Knowledge of Safety, Health, and Environment in Health Care Systems
}

\author{
Moahammad Baratchi' ${ }^{1}$, Nabiollah Mansouri², Aida Ahmadi ${ }^{*}$ \\ ${ }^{I}$ MSc, Department of HSE Management, Faculty of Environment and Energy, Science and Research Brand-Islamic Azad \\ University, Tehran, Iran \\ ${ }^{2}$ Professor, Department of HSE Management, Faculty of Environment and Energy, Science and Research Brand-Islamic Azad \\ University, Tehran, Iran \\ ${ }^{3}$ Assistant Professor, Department of HSE Management, Faculty of Environment and Energy, Science and Research Brand- \\ Islamic Azad University, Tehran, Iran \\ * Corresponding Author: Aida Ahmadi, Department of HSE Management, Faculty of Environment and Energy, Science and \\ Research Brand-Islamic Azad University, Tehran, Iran. Email: ahmadyaida@yahoo.com
}

Received: 09/04/2017

Accepted: 06/11/2017

\section{How to Cite this Article:}

Baratchi M, Mansouri N,

Ahmadi A. Introducing a

Combined Pattern to Empower

Risk Assessment Knowledge of

Safety, Health, and Environment in Health Care Systems. J Occup Hyg Eng. 2017; 4(3): 63-70. DOI:

\section{Abstract}

Background and Objective: Knowledge management is the optimal use of knowledge to achieve intended purposes. Given the type of activity of health care systems and their high health and safety risks, knowledge of health and safety executive (HSE) principles not only contributes to patient safety and health, but also enhances the health and safety of employees through raising awareness and using others' experiences.

Materials and Methods: In this study, a questionnaire with 11 key questions was developed. Then, to evaluate the validity and reliability of the primarily questionnaire, inter-method reliability and Cronbach's $\alpha$ coefficient were used. To analyze the data, binominal test was run in SPSS, version 22

Results: The validity of Relevancy, clarity, and Cronbach's alpha coefficient of the questionnaire were $92.72 \%, 91.18 \%$, and 0.7 , respectively. The results of binominal test showed that all the questions were accepted (above $75 \%$ for each) by the experts.

Conclusion: Although knowledge management is a niche area in Iran, it is important to apply new patterns in various aspects of the relevant activities such as HSE. It can be concluded that the introduced questionnaire is a valid and reliable tool that can be used in vocational processes.

Keywords: Content Validity; HSE; Knowledge Management; Questionnaire 
do:

ارائه الكوى تلفيق تواذمندسازى داشى ارزيابى ريسك ايمنى، بهداشت و محيط زيست

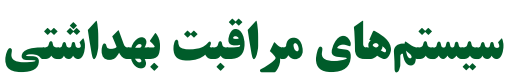

\author{
محمد براتجى'، نبى الله منصورى ‘، آيدا احمدى"*
}

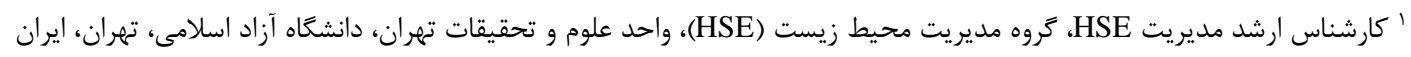

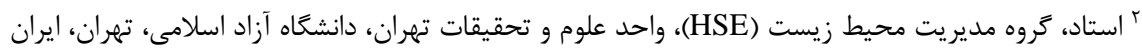

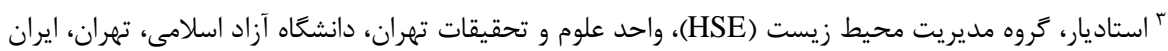

* نويسنده مسئول: آيدا احمدى، كروه مديريت محيط زيست (HSE)، واحد علوم و تحقيقات تهران، دانشًاه آزاد اسلامى، تهران، ايران. ايميل: ahmadyaida@yahoo.com

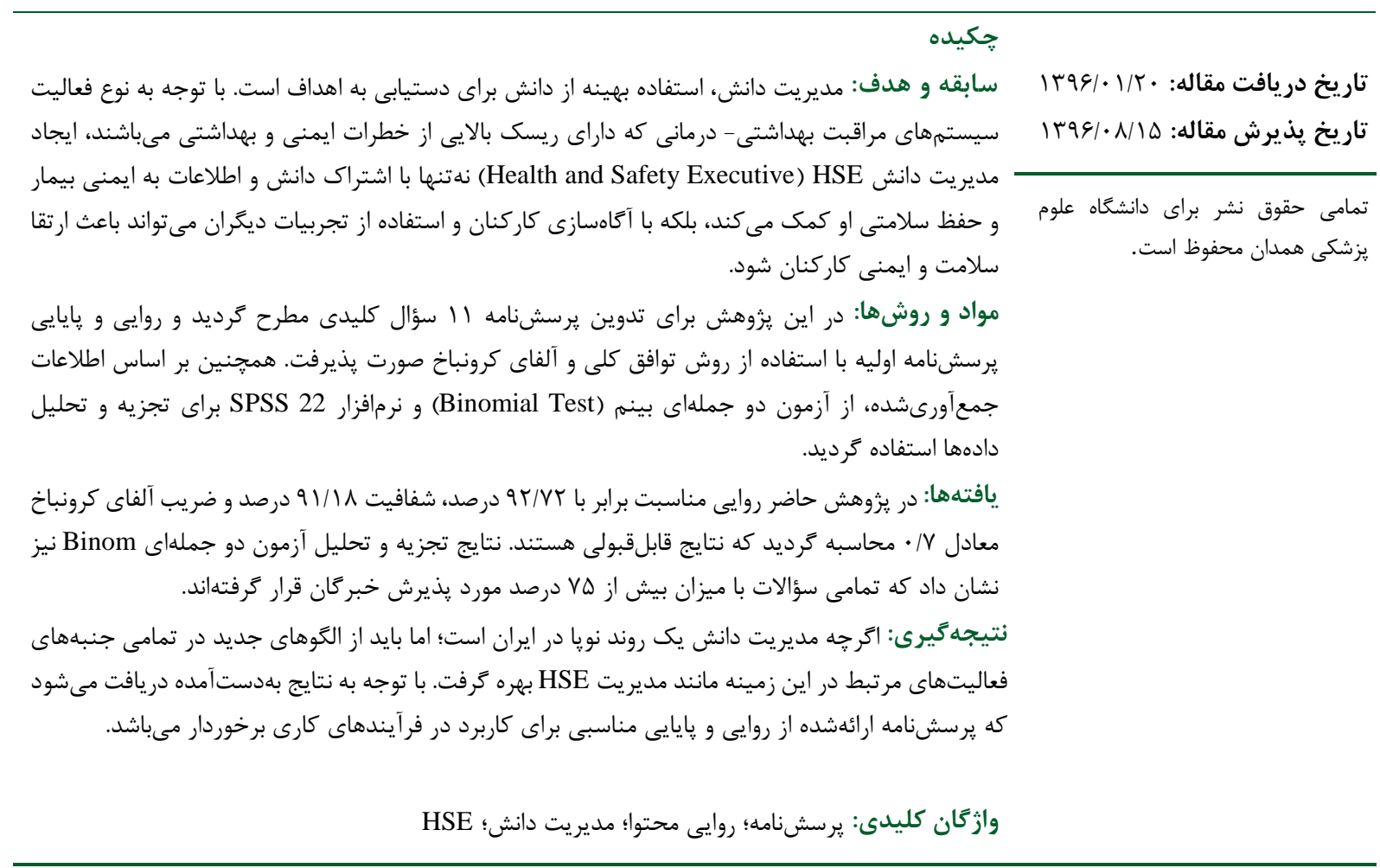

بهداشتى) را غربالكرى و سازماندهى نموده و به اطلاعات مفيد

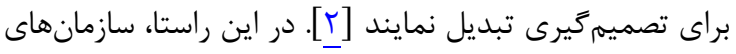

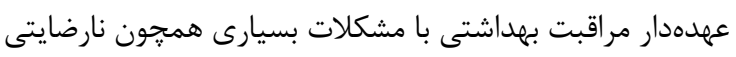

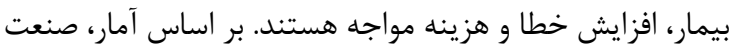

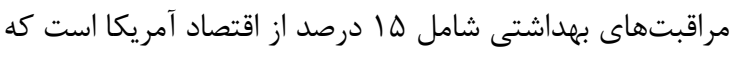

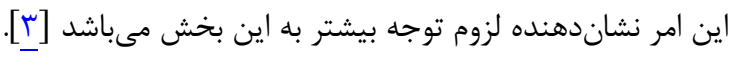

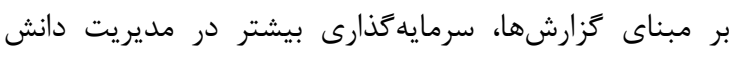

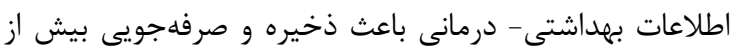

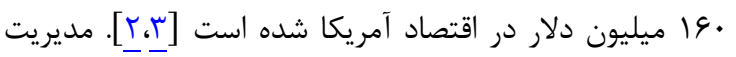

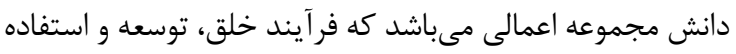

مقدمه براى حفظ و تداوم سيستمهاى مراقبت بهداشتى بايد انتقال

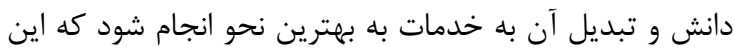

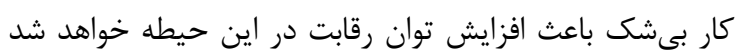

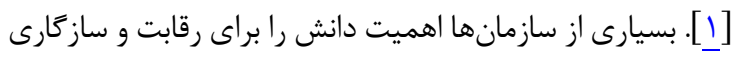

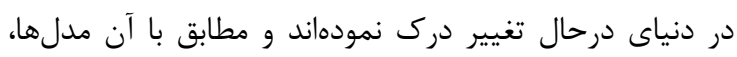

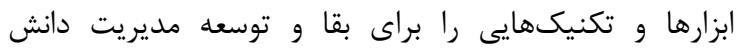

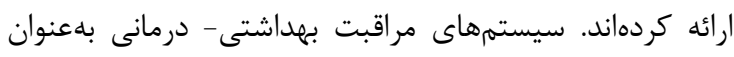

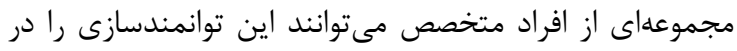

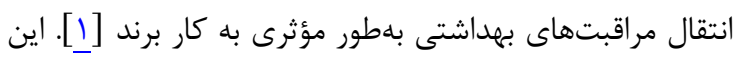

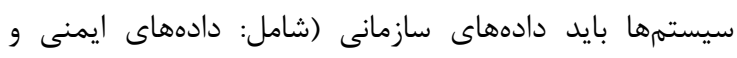


مديريت دانش براى توانمندسازى فعاليتها در زمينه ايمنى،

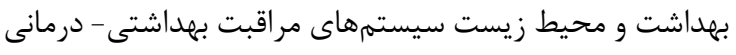

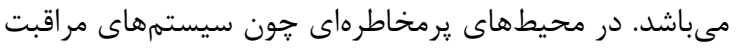

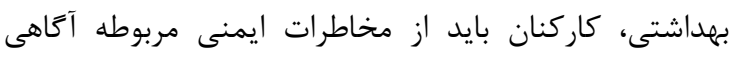

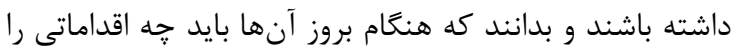

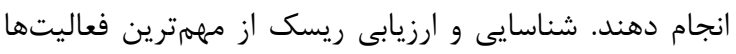

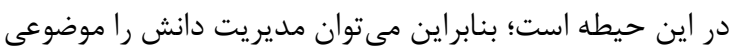

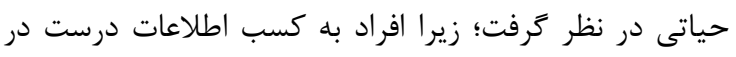

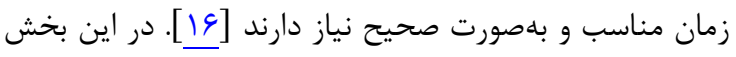
بلهطور اختصاصى به ارائه و معرفى الخو در اين زمينه يرداخته شده است.

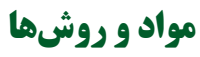

مطالعه و بررسى توصيفى از طريق تهيه و تدوين ترسشنامه

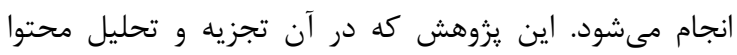

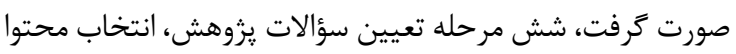

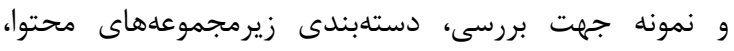
كدَذارى با استفاده از مقياس مناسب جهت تهون تكميل واحدهاى مورد بررسى، تست آزمايشى و تجزيه و تحليل دادههاى آمارى را راس

به شرح زير طى نموده است [IV]

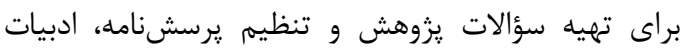

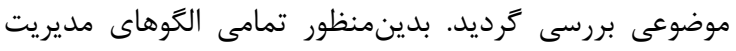

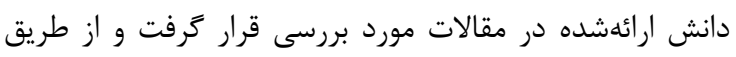

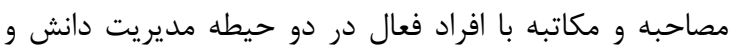

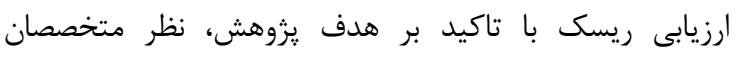

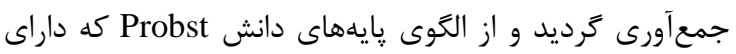

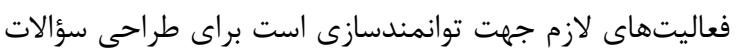

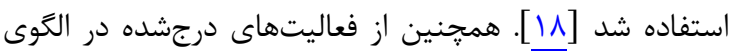

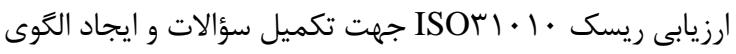

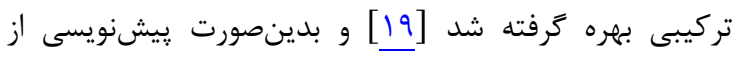
يرسشنامه يديد آمد.

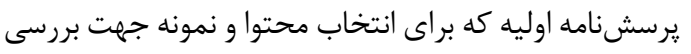

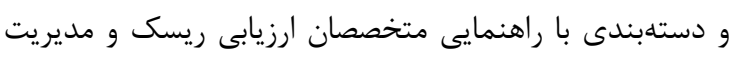

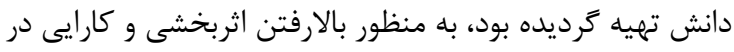

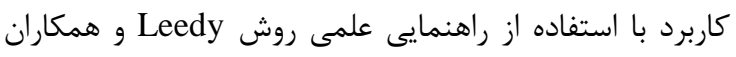

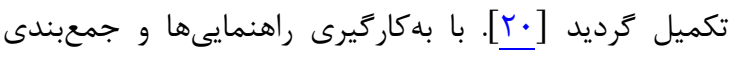

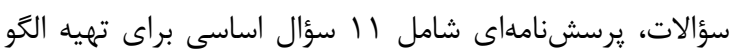

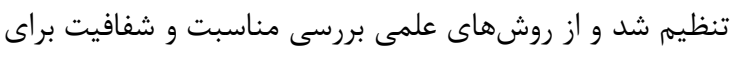
ارزيابى روايى محتوا استفاده گرديد.

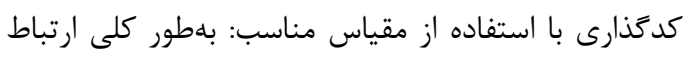

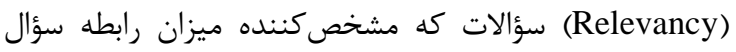

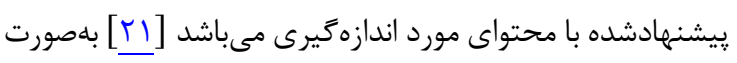

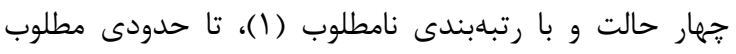

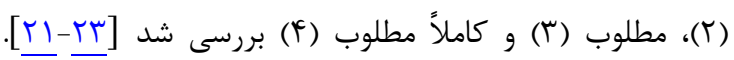

از دانش را هدايت كرده است و امروزه اهداف بيشتر سازمانها

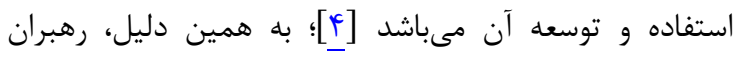

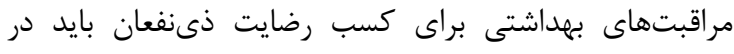
بسيارى از فاكتورهاى سازمانى از جمله مديريت داني دانش عملكرد

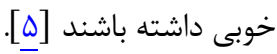

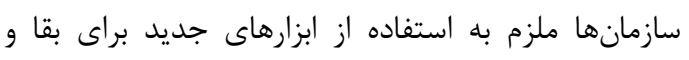
سرعتبخشيدن به بهبود نظام سازمانى خود هستند. مدير بريت

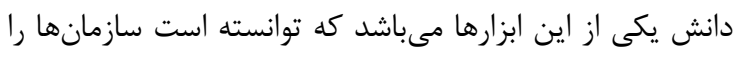

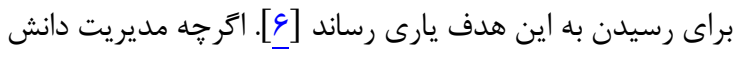

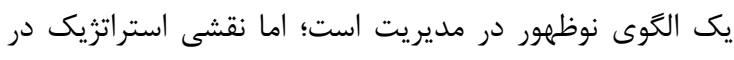

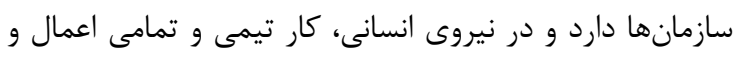

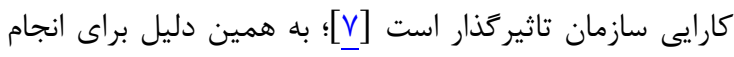
تصميم گيرى صحيح در سازمانها بايد از علم مديريت دانش بهان بهره

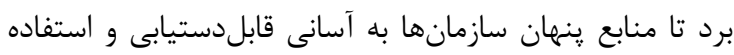
كردد [1] در سالهاى اخير مطالعات بسيارى در مورد مديريت دانش

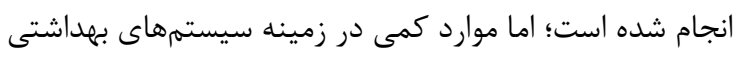

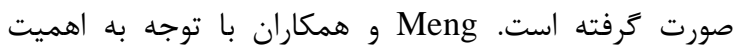

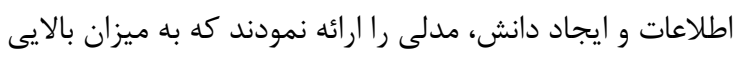

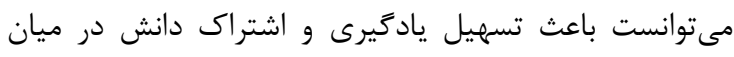

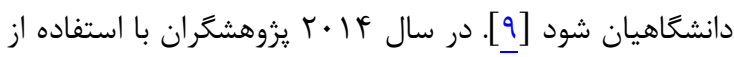

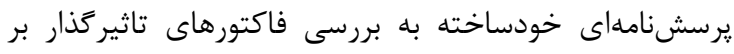

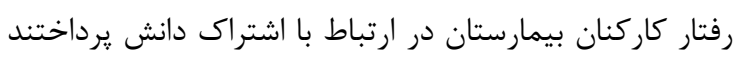

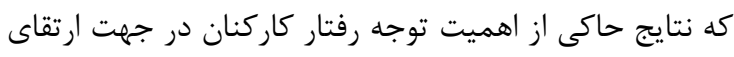

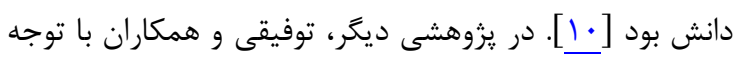

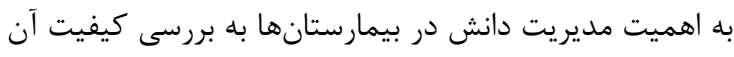

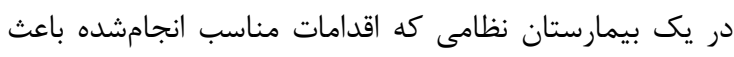

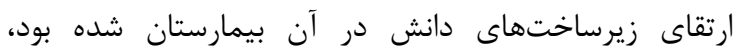

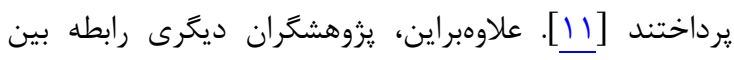

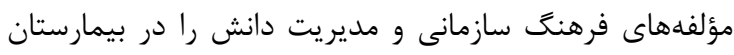

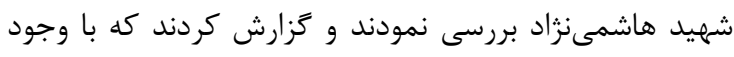

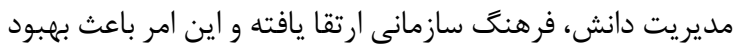

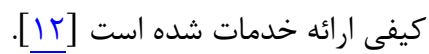

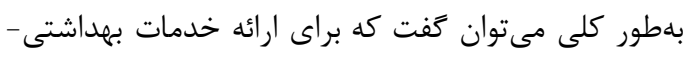

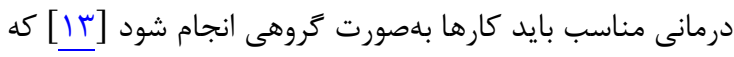

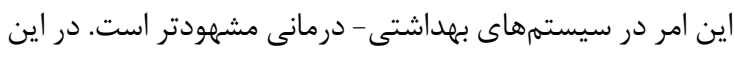

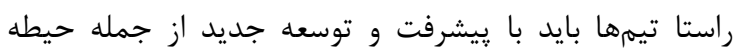

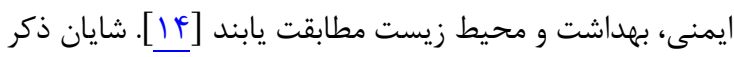

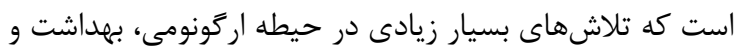

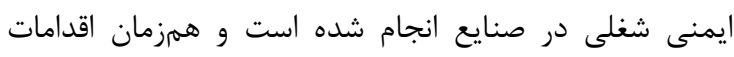
بسيارى در جهت مديريت دانش كاركنان و دانش ساختى دارنار

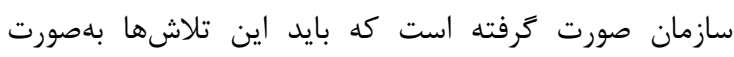
همزمان مديريت گردند [هان].

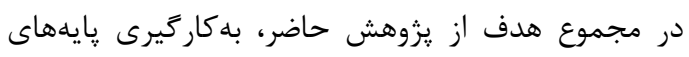


در آخرين مرحله براى تجزيه و تحليل دادههاى آمارى يس

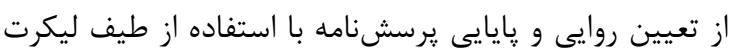

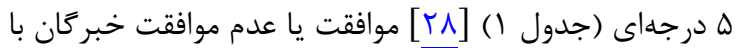

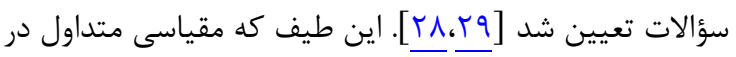

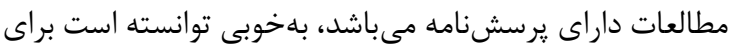

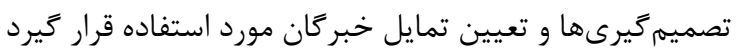

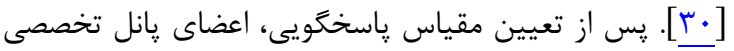

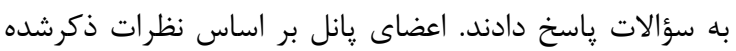

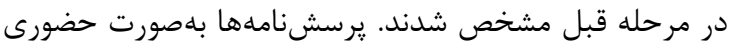

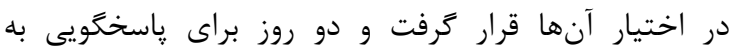

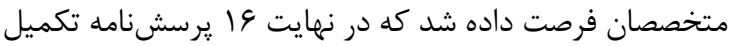

جدول ا: عبارات و اعداد طيف ليكرت ه درجهاى

\begin{tabular}{|c|c|c|c|c|}
\hline بسىاهميت & بىاهميت & متوسط & باهميت & باهيار \\
\hline 1 & $r$ & $r$ & $f$ & $\Delta$ \\
\hline
\end{tabular}

ويزگى افراد شركت كننده در جدول r ارائه شده است. براى

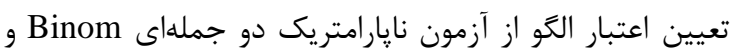

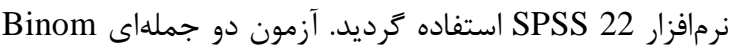

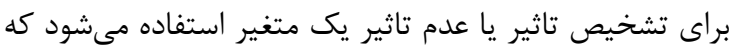

\begin{tabular}{|c|c|c|c|}
\hline فراوانى (درصد) & تعداد & كروه & متغير \\
\hline$\Delta \varphi$ & 9 & مرد & \multirow[b]{2}{*}{ جنس } \\
\hline FF & v & 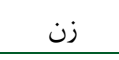 & \\
\hline rV & 4 & كارشناسى & \multirow{2}{*}{ تحصيلات } \\
\hline q & $1 \cdot$ & دكترى & \\
\hline
\end{tabular}

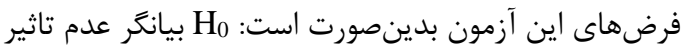

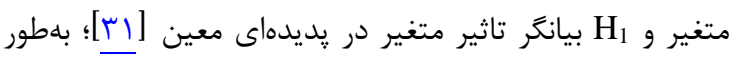

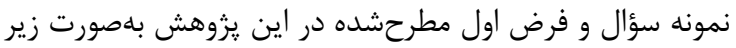

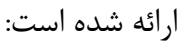

سؤال ا: ضرورت وجود عنصر هدفهاى دانش HSE در مرحله زمينهسازى الكو فرض صفر: وجود عنصر هدفهاى دانش HSE در مر مرحله زمينهسازى الكو ضرورى نمى باشد. فرض يك: وجود عنصر هدفهاى دانش HSE در بر مرحله زمينهسازى الكو ضرورى مىباشد.

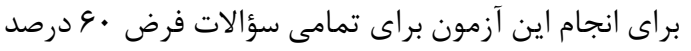

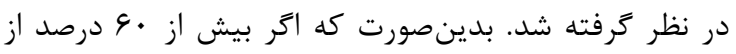

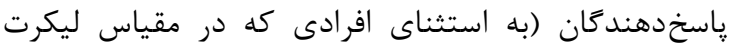

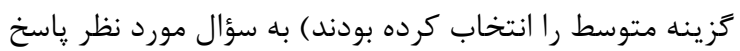

شفافيت (Clarity) محتواى سؤالات نيز كه مشخص كننده واضحبودن سؤالات از نظر نعارش و مفهوم آنها است نيز با همان

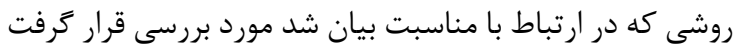

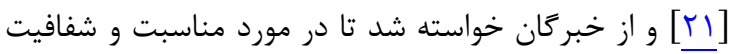

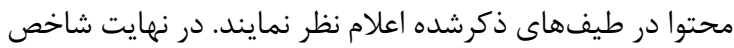

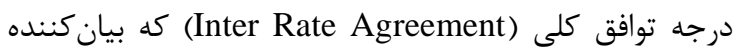
درجه توافق مشاهدهشده بين نظر متخصصان شركت كننده در

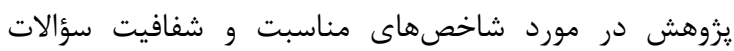

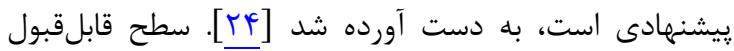

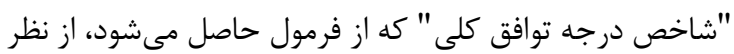

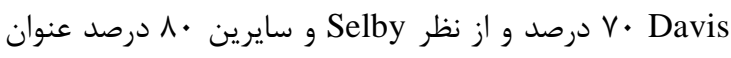

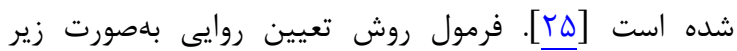

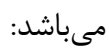

توافق كلى در مورد مناسبت (رويكرد كمتر محافظه كارانه) = تعداد توافق مشاهدهده بين متخصصان تقسيم بر تعداد كل موارد

اعضاى تعيين روايى، افرادى داراى تجربه يزوهش در تعيين

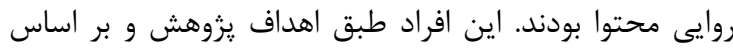

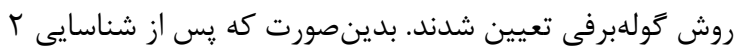

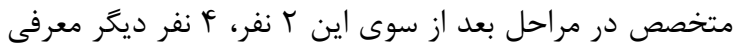

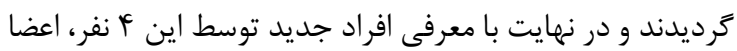

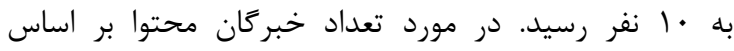

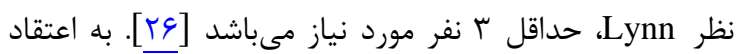

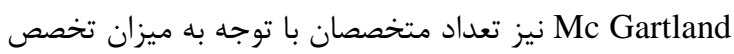

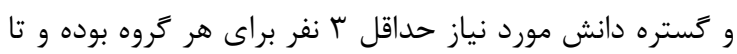

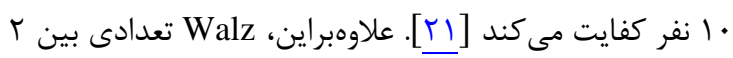

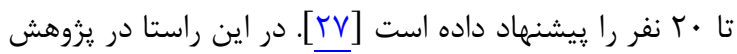

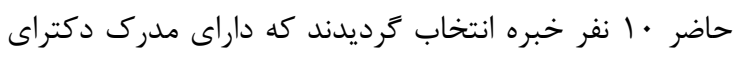

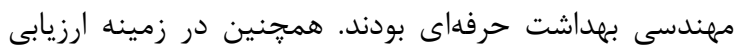

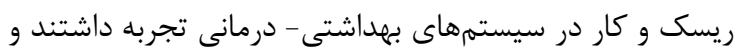

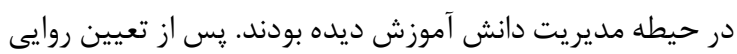

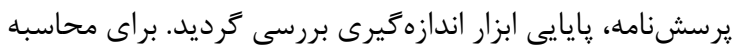

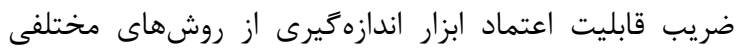

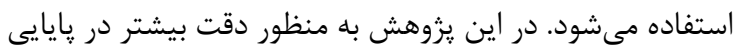

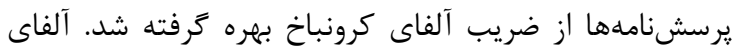

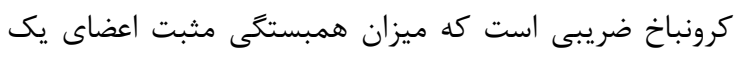

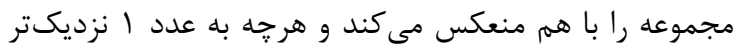

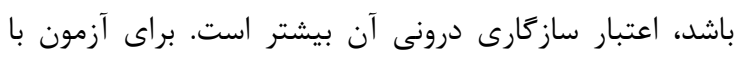

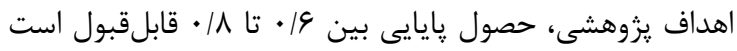

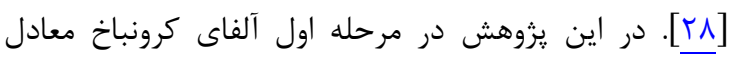

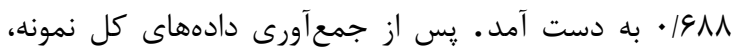

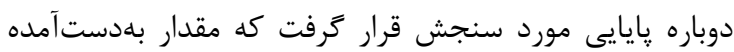

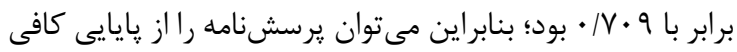
برخوردار دانست. 
جملهاى Binom مورد بررسى قرار گرفت. نمونه نتايج حاصل از SPSS دو مؤلفه اول اين آزمون در جدول عأ ارائه شده است.

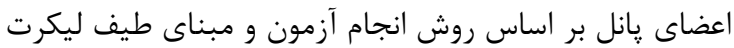

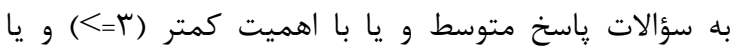

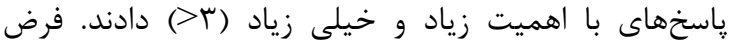

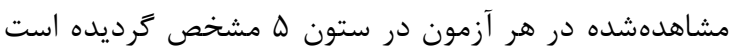

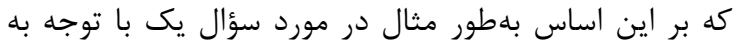

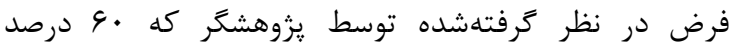

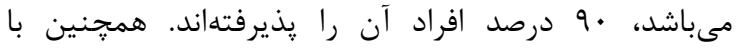

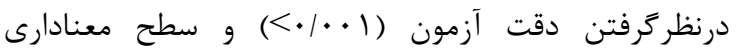
(ه •/•>)، سؤال مورد نظر بر اساس دون آزمون قابل إقبول بوده و مورد هذيرش خبر گان است.

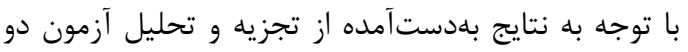

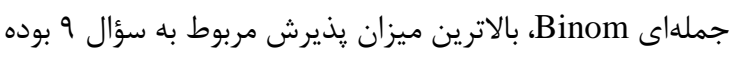

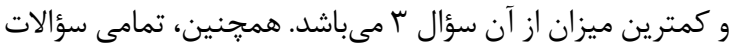

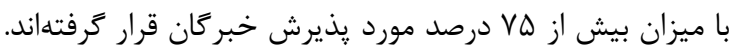

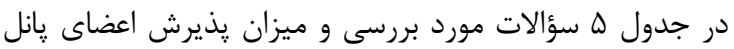

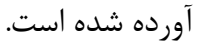

نمودار الكوى تركيبى بر اساس نتايج بلهدستآمده در شكل

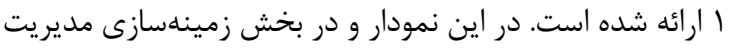

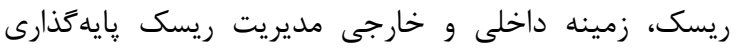

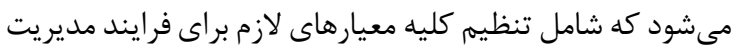

مثبت دهند (كزينههاى بااهميت و بسيار باهميت را انتخاب

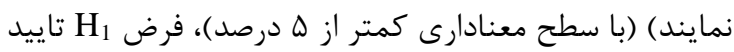

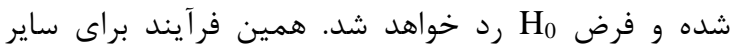

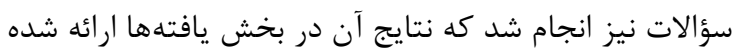

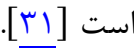

\section{كافته انا}

با توجه به مراحل شش كانه انجامشده در ارتباط با تعيين

روايى يرسشنامه و كسب نتيجه از يرسشنامه توزيعشده ميان

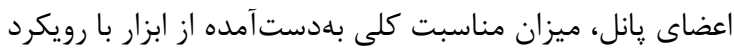

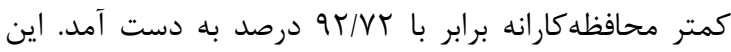

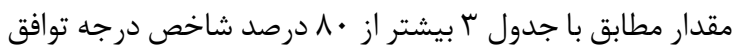

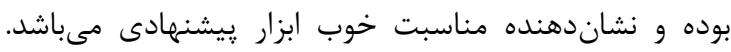

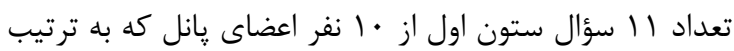

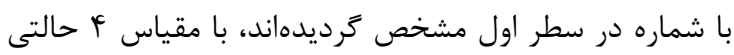

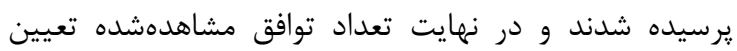
كرديدند. همجنين ميزان شفافيت كلى بهدست آمده ابزار برابر

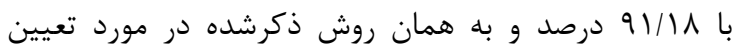

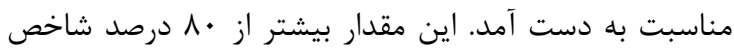

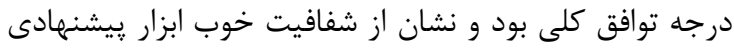
داشت. آزمون فرضيات: اعتبار پرسشنامه به كمك آزمون دو

جدول با: مقايسه مقادير شاخص مناسبت كلى يرسشنامه ل/ سؤالى بهدستآمده از رويكرد توافق كلى (كمتر محافظه كارانه)

\begin{tabular}{|c|c|c|c|c|c|c|c|c|c|c|c|c|c|}
\hline \multirow{2}{*}{ مناسبت كلى ابزار } & \multirow{2}{*}{ مناسبت } & \multirow{2}{*}{ تعداد توافق مشاهدهشده } & \multicolumn{10}{|c|}{ نمرات متخصصان به شاخص مناسبت هركدام از سؤالات } & \multirow{2}{*}{ سؤال } \\
\hline & & & $1 \cdot$ & 9 & $\wedge$ & $\checkmark$ & 9 & $\Delta$ & $f$ & r & $r$ & 1 & \\
\hline \multirow{11}{*}{ 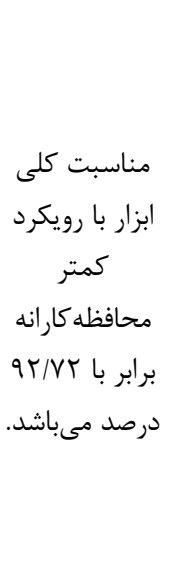 } & 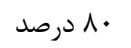 & $\wedge$ & $r$ & f & r & $r$ & $r$ & $r$ & r & $r$ & r & $r$ & 1 \\
\hline & 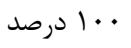 & 1. & $r$ & f & q & r & r & f & q & $r$ & r & f & r \\
\hline & 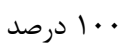 & 1. & $r$ & $r$ & f & $r$ & $r$ & r & q & $r$ & $r$ & $q^{f}$ & r \\
\hline & • ه درصد & 9 & r & $r$ & f & f & r & $r$ & $r$ & $r$ & $r$ & r & f \\
\hline & • ^ درصد & $\wedge$ & r & r & $r$ & r & i & r & $\mu$ & r & r & f & $\Delta$ \\
\hline & 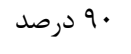 & 9 & r & r & $r$ & r & r & r & r & $r$ & r & $r$ & द \\
\hline & 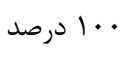 & $1 \cdot$ & $r$ & $r$ & r & $f$ & r & r & $r$ & r & r & q & $\checkmark$ \\
\hline & 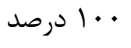 & 1. & $r$ & r & $\mu$ & r & i & r & $\mu$ & $r$ & $\mu$ & r & $\wedge$ \\
\hline & 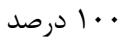 & $1 \cdot$ & $r$ & q & $r$ & r & q & $r$ & q & r & $\mu$ & q & 9 \\
\hline & 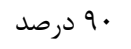 & 9 & $r$ & r & r & r & $\mu$ & r & f & r & $r$ & f & 1 . \\
\hline & • ج درصد & 9 & $r$ & $r$ & $r$ & $r$ & r & r & $r$ & $r$ & $r$ & $r$ & 11 \\
\hline
\end{tabular}

جدول fأ: نتايج تست دو جملهاى Binom

\begin{tabular}{|c|c|c|c|c|c|c|}
\hline دقت آزمون & فرض آزمون & فرض مشاهدهشده & تعداد & تروه & & \\
\hline \multirow{3}{*}{$<\cdot / \cdot \cdot 1$} & \multirow{3}{*}{$\cdot 19$} & $\cdot / 1$ & $r$ & $<=r$ & كروه 1 & \multirow{3}{*}{ سؤال ا } \\
\hline & & .19 & If & $>r$ & كروه r & \\
\hline & & $1 / \cdot$ & 19 & & 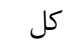 & \\
\hline \multirow{3}{*}{$\cdot 1 \cdot \cdot 1$} & \multirow{3}{*}{$\cdot / 9$} & $\cdot / r$ & r & $<_{=r}$ & كروه 1 & \multirow{3}{*}{ سؤال r } \\
\hline & & $\cdot 1 \mathrm{~A}$ & Ir & $>r$ & كروه r & \\
\hline & & $1 / \cdot$ & 19 & & كل & \\
\hline
\end{tabular}


جدول ه: نتيجه كلى ميزان پِيرش سؤالات الكو توسط خبركان

\begin{tabular}{|c|c|c|}
\hline درصد يذيرش توسط خبر گان & متن سؤال & شماره سؤال \\
\hline$\wedge \vee / \Delta$ & ضرورت وجود عنصر اهداف دانش HSE در مرحله زمينهسازى & 1 \\
\hline$\Lambda 1 / r \Delta$ & ضرورت وجود عنصر شناسايى دانش HSE در مرحله زمينهسازى & r \\
\hline$\checkmark \Delta$ & ضرورت وجود عنصر كسب دانش HSE در مرحله زمينهسازى & r \\
\hline$\wedge \vee / \Delta$ & ضرورت وجود عنصر استفاده از دانش HSE در مرحله ارزيابى ريسك & f \\
\hline$\Lambda 1 / r \Delta$ & ضرورت وجود عنصر نتخهدارى دانش HSE در مرحله ارزيابى ريسك & $\Delta$ \\
\hline$\Lambda 1 / T \Delta$ & ضرورت وجود عنصر تسهيم دانش HSE در مرحله ارتباطات و مشاوره & 4 \\
\hline$\Lambda 1 / \gamma \Delta$ & ضرورت وجود عنصر توسعه دانش HSE در مرحله كنترل و بازنترى & $\checkmark$ \\
\hline$\wedge \vee / \Delta$ & ضرورت وجود عنصر ارزيابى دانش HSE در مرحله كنترل و بازنگرى & $\wedge$ \\
\hline$q \mu / V \Delta$ & ضرورت وجود عنصر استفاده از دانش HSE در مرحله برخورد با ريسك & 9 \\
\hline$\wedge \vee / \Delta$ & جامع و كاملبودن الكو & $1 \cdot$ \\
\hline$\wedge \vee / \Delta$ & قابليت به كاركيرى الكَو براى سيستمهاى خدمات بهداشتى - درمانى & 11 \\
\hline
\end{tabular}

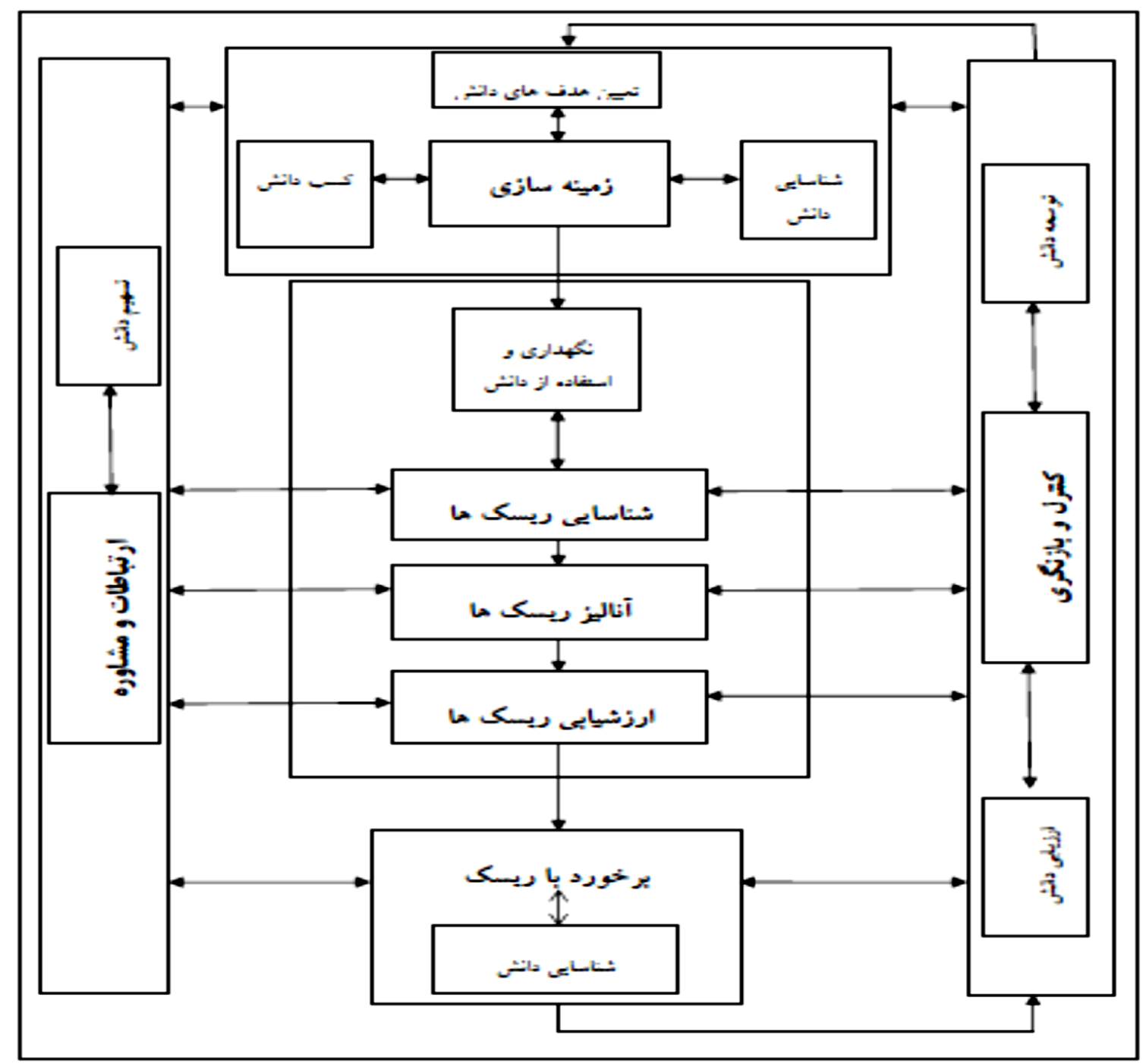

شكل ا: الكوى بيشنهادى توانمندسازى دانش ارزيابى ريسك ايمنى، بهداشت و محيط زيست

موردقبول قرار كرفته است. در اين راستا تسهيم دانش در

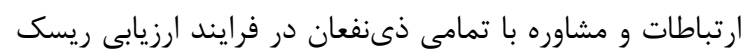

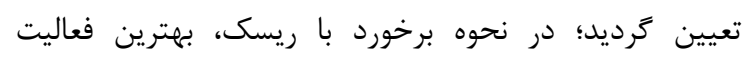

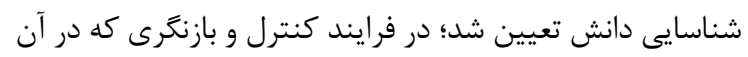

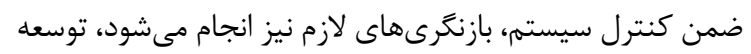

ريسك مانند تعيين معيارهاى ارزيابى مخاطره مىباشد. در اين

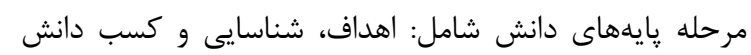
بلهعنوان ابزارهاى توانمندساز يذيرفته شدند.

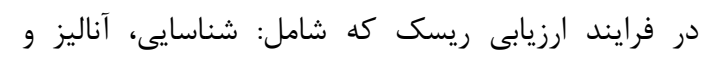

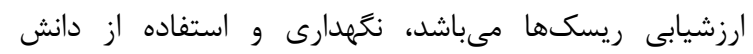


همانند زعفريان و همكاران كه الخويى شش مرحلهاى بالـ مؤلفهاى تعيين اهداف دانش، فناورى اطلاعات، شناسايى

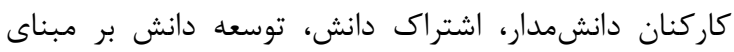

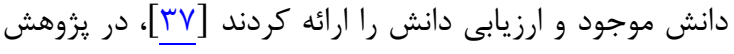

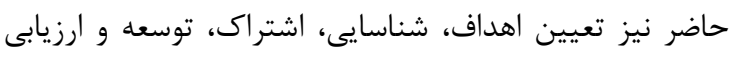
دانش بهعنوان بخشهاى كليدى در الكو معرفى شدند. در اين

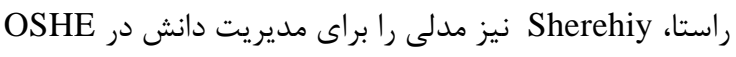
(Occupational Safety, Health, and Environment) معرفى كرد. اين مدل شامل: سيستمهاى دانش سازمانى،

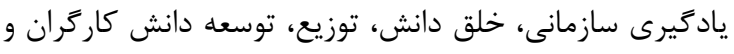

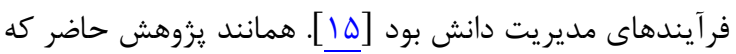

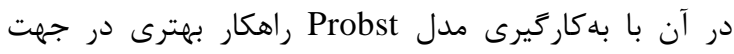

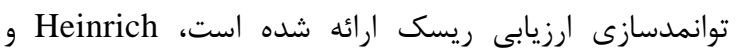
همكاران نيز با به كاركيرى جهار جوب Probst توانستند رئد راهكار مناسبى را براى كمك به توليد اطلاعات منظم و خدمار خدمات بهات

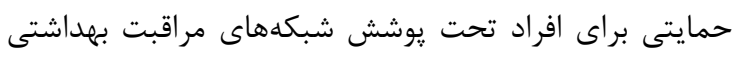

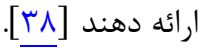

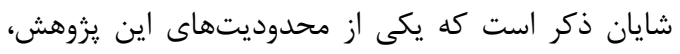
احتمال عدم احاطه كامل ياسخدهند

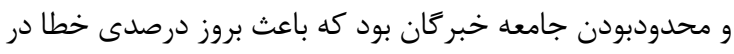
ياسخها مىشود.

نتيجه كيرى

نتايج حاصل از اين بررسى، ارائه الگويى براى مديريت دانش HSE سيستمهاى مراقبت بهداشتى است كه به كمك آنى

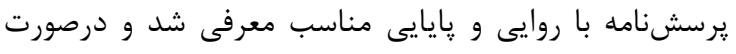

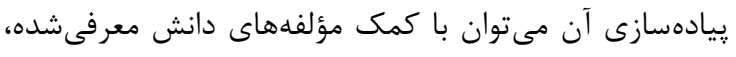

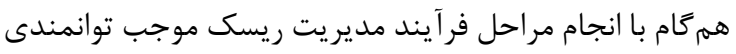

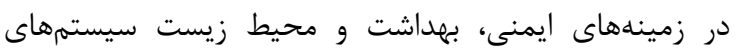

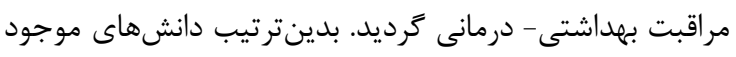

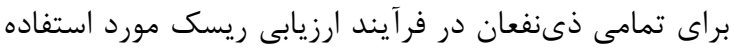

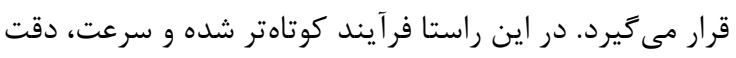

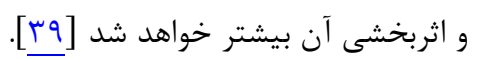

\section{تشكر و قدردانى}

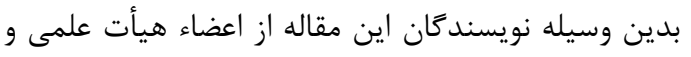

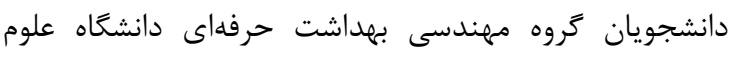
يزشكى تهران كه در اين تحقيق همكارى نمودند تشكر فئن مى نمايند.

\section{REFERENCES}

1. Van Beveren J. Does health care for knowledge management? J Knowl Manag. 2003;7(1):90-5. DOI:10.1108/136732 $\underline{70310463644}$

2. Pavia L. The era of knowledge in health care. Health Care
Strateg Manage. 2001;19(2):12-3. PMID: 11219266

3. Gowen III CR, Henagan SC, McFadden KL. Knowledge

مديريت دانش داراى ارزش و كاربرد فراوانى در صنعت

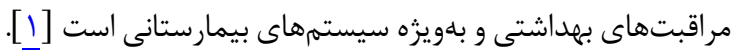

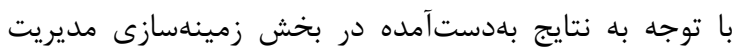

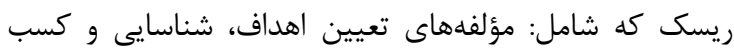
دانش مىباشد، اهداف اصلى سازمان مشخص مؤه شده و و تمامى

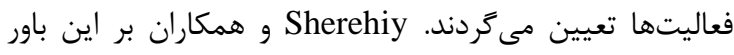

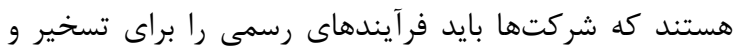

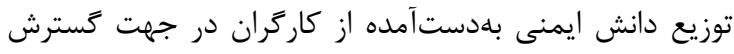

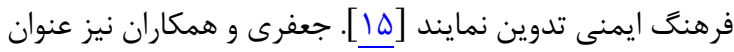

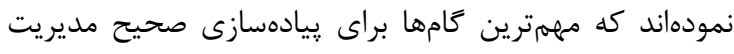

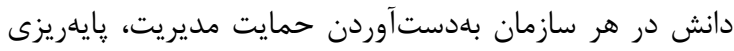

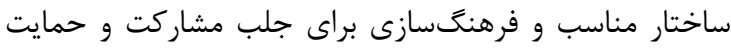

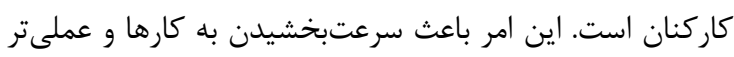

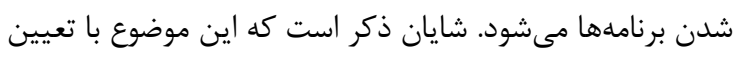

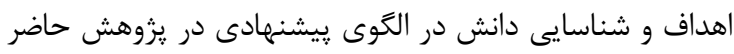

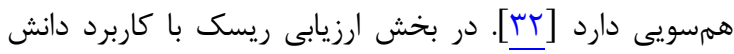

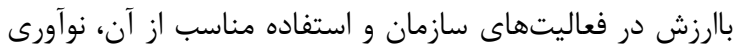

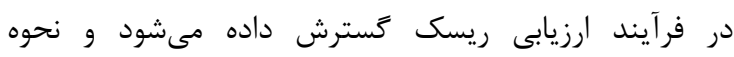
مستندسازى مشخص مى

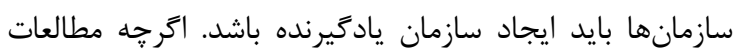
بسيارى در ارتباط با مديريت دانش انجام شده است؛ اما تنها باديا

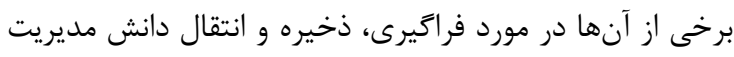

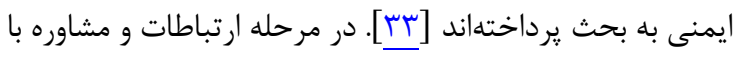

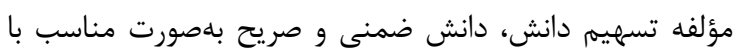

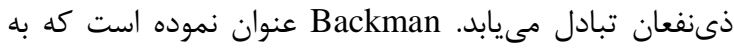

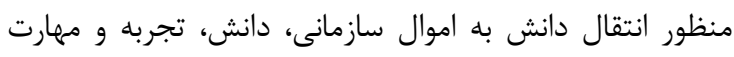

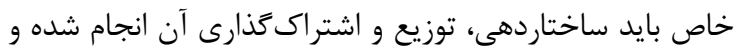

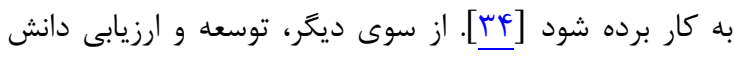
باعث ايجاد ايدهها، گسترش دانش و بازخورد مناسب درد در درد مرحله

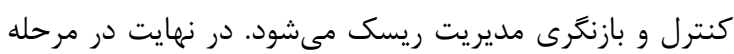

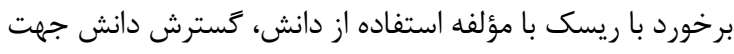

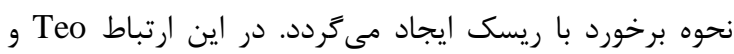

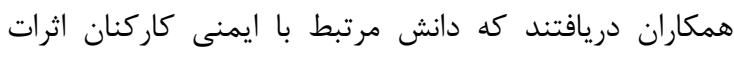

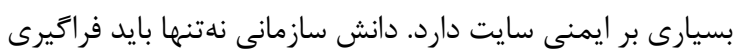

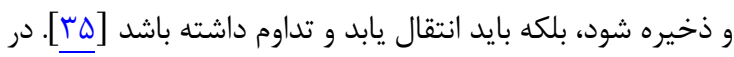

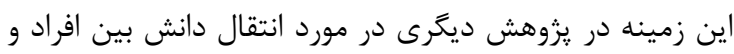

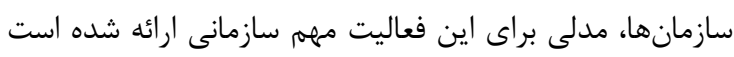
كه هدف آن انتقال دانش ايمنى و بهداشت بين افراد و و سازمان 
management as a mediator for the efficacy of transformational leadership and quality management initiatives in US health care. Health Care Manag Rev. 2009;34(2):129-40. DOI: 10.1097/HMR.0b013e31819e9169

4. Ghorbanizadeh V, Mohammadi MY. Knowledge management model in police organization. Quart J Police Manag Res. 2012;7(3):328-45. [Persian]

5. Alexander JA, Hearld LR, Jiang HJ, Fraser I. Increasing the relevance of research to health care managers: hospital CEO imperatives for improving quality and lowering costs. Health Care Manage Rev. 2007;32(2):150-9. PMID: 17438398 DOI: 10.1097/01.HMR.0000267792.09686.e3

6. Ahmady GA, Nikooravesh A, Mehrpour M. Effect of organizational culture on knowledge management based on Denison model. Proc Soc Behav Sci. 2016;230:387-95. DOI:10.1016/j.sbspro.2016.09.049

7. Torabi MH, Kyani A, Falakinia H. An investigation of the impact of knowledge management on human resource performance in management of keshavarzi bank branches in Tehran. Proc Soc Behav Sci. 2016;230:471-81. DOI:10.1016/j.sbspro.2016.09.059

8. Nikookar GH, Hakim A. Strategic alignment of knowledge management in organization. Maneg Res Iran. 2012;16(1):167-87. [Persian]

9. Cui M, Yang S, Yu T, Yang C, Gao Y, Zhu H. Expert knowledge maps for knowledge management: a case study in Traditional Chinese Medicine research. J Tradit Chin Med. 2013;33(5):698-702. PMID: 24660599

10. Lee HS, Hong SA. Factors affecting hospital employees' knowledge sharing intention and behavior, and innovation behavior. Osong Public Health Res Perspect. 2014;5(3):14855. PMID: 25180147 DOI: 10.1016/j.phrp.2014.04.006

11. Sh T, Fallah MS, Khajeh Azad M. Quality evaluation of knowledge management in a military hospital based on the Baldrige excellence model. J Mil Med. 2012;13(4):213-6. [Persian]

12. Sadeghi A, Jafari $H$, Khodayari R, Pakdaman M, Mohammadi R, Ahadi NB. A case study: the association between organizational culture with management knowledge in hasheminezhad hospital-Tehran. Hospital. 2011;10(2):1-8. [Persian]

13. West MA, Wallace M. Innovation in health care teams. Eur $J$ Soc Psychol. 1991;21(4):303-15. DOI: 10.1002/ejsp. 2420210404

14. Kessel M, Kratzer J, Schultz C. Psychological safety, knowledge sharing, and creative performance in healthcare teams. Creativ Innovat Manage. 2012;21(2):147-57. DOI: 10.1111/j.1467-8691.2012.00635.x

15. Sherehiy B, Karwowski W. Knowledge management for occupational safety, health, and ergonomics. Hum Fact Ergon Manufact Serv Indust. 2006;16(3):309-19. DOI: 10.1002/hfm.20054

16. Boy G, Barnard Y. Knowledge management in the design of safety-critical systems. Hershey. PA, USA: Encyclopedia of Knowledge Management; 2005.

17. Prasad BD. Content analysis. New Delhi: Research Methods for Social Work; 2008. P. 5.

18. Probst GJ. Practical knowledge management: a model that works. Massachusetts: Prism-Cambridge; 1998. P. 17-30.

19. The International Electrotechnical Commission (IEC). Risk management-risk assessment techniques. Final Draft (IEC/FDIS 31010 ). New York: International Standard; 2009. P. 92.

20. Leedy PD. Practical research: planning and design. Basingstoke, UK: Macmillan; 1993.
21. Rubio DM, Berg-Weger M, Tebb SS, Lee ES, Rauch S. Objectifying content validity: Conducting a content validity study in social work research. Soc Work Res. 2003;27(2):94104. DOI:10.1093/swr/27.2.94

22. Grant JS, Davis LL. Selection and use of content experts for instrument development. Res Nurs Health. 1997;20(3):26974. PMID: 9179180

23. Schutz AL, Counte MA, Meurer S. Development of a patient safety culture measurement tool for ambulatory health care settings: analysis of content validity. Health Care Manag Sci. 2007;10(2):139-49. PMID: 17608055

24. Polit DF, Beck CT. The content validity index: are you sure you know what's being reported? Critique and recommendations. Res Nurs Health. 2006;29(5):489-97. PMID: 16977646 DOI: 10.1002/nur.20147

25. Abdollahpour I, Nedjat S, Noroozian M, Majdzadeh R. Performing content validation process in development of questionnaires. Iran J Epidemiol. 2011;6(4):66-74. [Persian]

26. Lynn MR. Determination and quantification of content validity. Nurs Res. 1986;35(6):382-6.

27. Waltz CF, Strickland OL, Lenz ER. Measurement in nursing and health research. New York: Springer Publishing Company; 2010.

28. Gliem JA, Gliem RR. Calculating, interpreting, and reporting Cronbach's alpha reliability coefficient for Likert-type scales. Midwest Research-to-Practice Conference in Adult, Continuing, and Community Education, The Midwest United States; 2003

29. Barua A. Methods for decision-making in survey questionnaires based on Likert scale. J Asian Sci Res. 2013;3(1):35-8.

30. Likert R. A technique for the measurement of attitudes. Washington, D.C.: Archives of Psychology; 1932.

31. Momeni M. Statistical analysis with SPSS. Tehran: Sanaei Publication; 2015. [Persian]

32. Jafari M, Akhavan P, Mortezaei A. Comparing models of knowledge management and introducing a combined model. Tomorrow Manage. 2009;8(21):15. [Persian]

33. Hallowell MR. Safety-knowledge management in American construction organizations. $J$ Manag Engin. 2011; 28(2):203-11.

34. Hadikusumo BH, Rowlinson S. Capturing safety knowledge using design-for-safety-process tool. J Construct Engin Manag. 2004;130(2):281-9.

35. Teo EA, Ling FY, Chong AF. Framework for project managers to manage construction safety. Int J Project Manag. 2005;23(4):329-41. DOI:10.1016/j.ijproman.2004.09.001

36. Roy M, Parent R, Desmarais L. Knowledge networking: a strategy to improve workplace health and safety knowledge transfer. Electron J Knowl Manag. 2003;1(2):159-66.

37. Zafarian R, Esmaeilzadeh M, Shahi N. Implementation of a new model of knowledge management in SMEs case study. $J$ Entrepreneurship Dev. 2009;1(2):75-102. [Persian]

38. Heinrich S, Uribe FL, Roes M, Hoffmann W, Thyrian JR, Wolf-Ostermann $\mathrm{K}$, et al. Knowledge management in dementia care networks: a qualitative analysis of successful information and support strategies for people with dementia living at home and their family caregivers. Public Health. 2016;131:40-8. DOI:10.1016/j.puhe.2015.10.021

39. Baratchi M. Risk assessessing and introducing HSE knowledge management pattern in selected Hospitals of Tehran. [Master Thesis]. Tehran: Islamic Azad University, Science and Research Branch, Faculty of Environment and Energy-Department of Environmental Management; 2017. [Persian] 